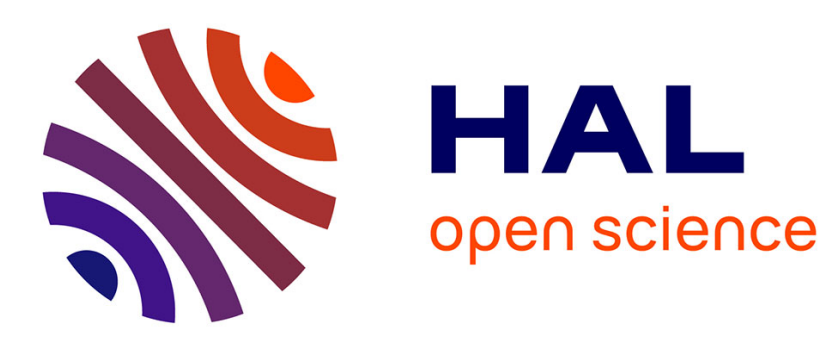

\title{
A Semi-analytical Method to Model Effective SINR Spatial Distribution in WiMAX Networks
}

\author{
M. Maqbool, M. Coupechoux, P. Godlewski
}

\section{To cite this version:}

M. Maqbool, M. Coupechoux, P. Godlewski. A Semi-analytical Method to Model Effective SINR Spatial Distribution in WiMAX Networks. IEEE Sarnoff Symposium, Mar 2009, Princeton, United States. pp.1-5. hal-01547163

\section{HAL Id: hal-01547163 https://hal-imt.archives-ouvertes.fr/hal-01547163}

Submitted on 26 Jun 2017

HAL is a multi-disciplinary open access archive for the deposit and dissemination of scientific research documents, whether they are published or not. The documents may come from teaching and research institutions in France or abroad, or from public or private research centers.
L'archive ouverte pluridisciplinaire HAL, est destinée au dépôt et à la diffusion de documents scientifiques de niveau recherche, publiés ou non, émanant des établissements d'enseignement et de recherche français ou étrangers, des laboratoires publics ou privés. 


\title{
A Semi-analytical Method to Model Effective SINR Spatial Distribution in WiMAX Networks
}

\author{
Masood Maqbool, Marceau Coupechoux, Philippe Godlewski \\ TELECOM ParisTech \& CNRS LTCI \\ 46, rue Barrault 75013 Paris, France \\ Email: \{firstname.lastname\}@ telecom-paristech.fr
}

\begin{abstract}
The stationary probabilities of different modulation and coding schemes (MCS) are required for dimensioning an OFDMA based network. In this paper, we introduce a semianalytical approach to find out these stationary probabilities for a WiMAX network in downlink (DL) with users served by the best base station (BS). Using Monte Carlo simulations, we find the spatial distributions of effective signal to interferenceplus-noise ratio $\left(S I N R_{e f f}\right)$ for different values of shadowing standard deviation $\left(\sigma_{S H}\right)$. With the help of distribution fit, we show that generalized extreme value (GEV) distribution provides a good fit for different frequency reuse schemes. Furthermore, by applying curve fitting, we demonstrate that the parameters of GEV distributions, as a function of $\sigma_{S H}$ values, can be expressed using polynomials. These polynomial can then be used off-line (in place of time consuming simulations) to find out GEV cumulative distribution function (CDF), and hence the stationary probabilities of MCS, for any desired value of $\sigma_{S H}$. We further show that these polynomials can be used for other cell configurations with acceptable deviation and significant time saving.
\end{abstract}

Keywords: OFDMA, PUSC, IEEE 802.16e, WiMAX, $S I N R_{e f f}$, MIC, best base station.

\section{INTRODUCTION}

WiMAX, a broadband wireless access technology, is based on IEEE standard 802.16-2005 [1]. Physical layer of WiMAX is characterized by orthogonal frequency division multiple access (OFDMA). With OFDM, available spectrum is split into a number of parallel orthogonal narrowband subcarriers. These subcarriers are grouped together to form subchannels. The distribution of subcarriers to subchannels is done using three major permutation methods called: partial usage of subchannels (PUSC), full usage of subchannels (FUSC) and adaptive modulation and coding (AMC). The subcarriers in a subchannel for first two methods are distributed throughout the available spectrum while these are contiguous in case of AMC.

A slot, the basic and minimum resource unit of a WiMAX system, occupies place both in time (OFDM symbols) and frequency (subchannel) domains thus introducing both the time and frequency multiple access. One of the important features of IEEE 802.16 based network is assignment of MCS type to a user depending upon its $S I N R_{\text {eff }}$ (cf. section III-B) value. Though the number of subcarriers possessed by a slot is fixed (i.e., forty eight), the number of bits it can transfer depends upon the MCS type used by the user. Therefore, cell throughput depends upon the probabilities of the possible MCS types. These MCS probabilities can be used in traffic analysis to obtain network dimensioning parameters (cf. section II). Since each MCS type is characterized by a $S I N R_{\text {eff }}$ threshold value, we require CDF of $S I N R_{\text {eff }}$ spatial distribution. Therefore an efficient way to obtain this CDF is always desired.

The study of SINR statistics in cellular environment is not recent. For examples, analytical/semi-analytical modeling of interference for mobile radio networks employing code division multiple access (CDMA) is given in [2]-[4]. However, the analysis carried out with single carrier in the physical layer can not be applied to multi carrier OFDMA based networks since the latter offers frequency diversity. System level simulations (SLS) have been used in [5]-[10] to find out percentage of MCS for an IEEE 802.16 based networks. The drawback of methods based purely on simulations is the excessive time consumption. In [11], an analytical method to calculate MCS probabilities and hence throughput in AMC mode of WiMAX has been proposed. However, the analysis does not take into account the shadowing effect. The authors of [12] present a semi-analytical method to calculate outage probabilities in OFDMA network (with no consideration of WiMAX specifications). In [13], an analytical calculation of symbol error rate for different MCS types is presented. To calculate symbol error, authors have not taken $S I N R_{\text {eff }}$ into account. In short, a method is required by which modeling of $S I N R_{e f f}$ statistics in WiMAX networks can be carried out more efficiently.

In this paper, we propose a semi-analytical method to find out stationary probabilities of different MCS types for a mobile WiMAX network that can substitute a number of simulations. We start with Monte Carlo simulations and find out spatial distributions of $S I N R_{e f f}$ for some integral values $\sigma_{S H}$. It is shown that the probability density functions (PDF) of $S I N R_{e f f}$ can be approximated by GEV distributions [14]. We exhibit that GEV distributions' parameters can be expressed in terms of $\sigma_{S H}$ using polynomial. Instead of simulations, these polynomials can then be used to find out GEV distribution, and hence MCS probabilities, for any desired value of $\sigma_{S H}$ in the above range. Furthermore, we demonstrate the applicability of these polynomials for different values of cell range $R$ and BS transmission power $P_{T x}$ and discuss the time efficiency offered.

Rest of the paper is organized as follows: section II gives a 


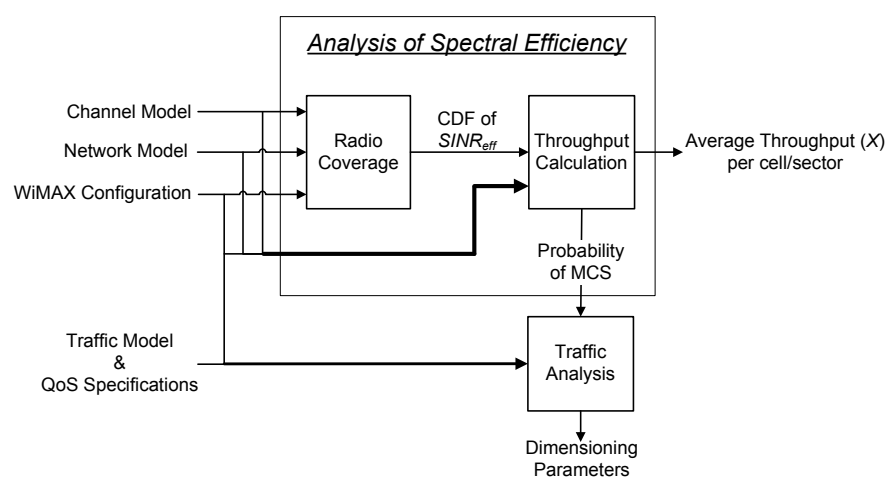

Fig. 1. System overview.

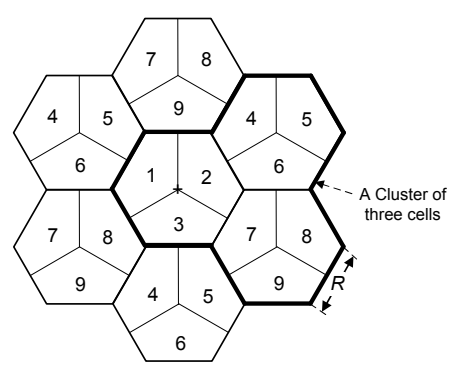

Fig. 2. Frequency Reuse Pattern $3 \times 3 \times 3$ ( $R$ is the cell range).

brief description of network dimensioning study and relative details of IEEE $802.16 \mathrm{e}$ system. In section III, SIN $R_{e f f}$ computation is discussed. The proposed semi-analytical method is described in section IV. Numerical results are presented in section V and finally section VI discusses the conclusion of this paper.

\section{Network Dimensioning}

The study of network dimensioning for mobile WiMAX networks can be divided into different components. As shown in Fig. 1, we classify it into three components: Radio Coverage, Throughput Calculation and Traffic Analysis. The work carried out in this paper focuses on Radio Coverage and Throughput Calculation blocks.

\section{A. Radio Coverage}

The input parameters to this block are: channel model, network model and WiMAX configuration. These parameters are mainly based on [15]. The output of this block is CDF of $S I N R_{\text {eff }}$ which can be obtained through Monte Carlo simulations. The disadvantage of simulation approach is excessive time consumption. In this paper, we intend to substitute the simulation approach by a semi-analytical method.

We have considered distributed subcarrier permutation type PUSC in our simulations. The analysis equally holds for subcarrier permutation type FUSC. Because of space limitation, we only discuss reuse type $3 \times 3 \times 3$ (shown in Fig. 2) in this paper. However, method was also verified for five other reuse types: $1 \times 1 \times 1,1 \times 3 \times 1,1 \times 3 \times 3,3 \times 1 \times 1$ and $3 \times 3 \times 1$ and results can be referred from [16]. The above mentioned six reuse types have been proposed in [15] for WiMAX networks.
TABLE I

THRESHOLD OF $S I N R_{e f f}$ VALUES FOR SIX MCS TYPES [17].

\begin{tabular}{|c|c|c|c|}
\hline Index & MCS & bits per slot $m_{k}$ & SINR $_{\text {eff }}$ [dB] \\
\hline \hline 0 & Outage & 0 & $<2.9$ \\
\hline 1 & QPSK 1/2 & 48 & 2.9 \\
\hline 2 & QPSK 3/4 & 72 & 6.3 \\
\hline 3 & 16QAM 1/2 & 96 & 8.6 \\
\hline 4 & 16QAM 3/4 & 144 & 12.7 \\
\hline 5 & 64QAM 2/3 & 192 & 16.9 \\
\hline 6 & 64QAM 3/4 & 216 & 18 \\
\hline
\end{tabular}

\section{B. Throughput Calculation}

Once the Radio Coverage block furnishes the CDF of $S I N R_{e f f}$, we require thresholds values of different MCS types to calculate MCS probabilities. Six different MCS types have been considered in our simulation model: QPSK-1/2 (the most robust), QPSK-3/4, 16QAM-1/2, 64QAM-2/3 and 64QAM-3/4 (for the best radio conditions). SIN $R_{\text {eff }}$ threshold values for MCS types are given in Tab. I and have been referred from [17]. If $S I N R_{\text {eff }}$ of a mobile station (MS) is less than the threshold of the most robust MCS (i.e., less than $2.9 \mathrm{~dB}$ ), it can neither receive nor transmit anything and is said to be in outage. We call outage as MCS type 0 .

Using the probabilities of MCS, the average cell throughput $X$ [bps] in DL is given as:

$$
X=\frac{N_{S}}{T_{F}} \sum_{k=1}^{K} m_{k} p_{k},
$$

where $K$ represents the total number of considered MCS types. The other two variables, $p_{k}$ and $m_{k}$, are respectively the probability and bits per slot for MCS type $k, N_{S}$ is the number of slots in DL sub-frame in a cell (i.e., per three sectors) and $T_{F}$ is the duration of TDD (time division duplex) frame.

Total bandwidth in our simulator has been set to $10 \mathrm{MHz}$. The number of OFDM symbols in a WiMAX TDD frame is considered to be 47 [15]. We assume two symbols fixed for common channel transmissions. The rest of 45 symbols are partitioned between DL and uplink sub-frames with DL part assuming two third of the symbols. Considering the sym$\mathrm{bol} / \mathrm{bandwidth}$ information, reuse type $3 \times 3 \times 3$ and permutation type PUSC, there are $N_{S}=450 \times 1 / 3$ slots in DL sub-frame, where $1 / 3$ appears because of reuse type.

\section{Traffic Analysis}

From Throughput Calculation, we get the available sector/cell throughput. However, utilization of this throughput depends upon the scheduling of different types of incoming traffic. A number of traffic types, characterized by application and QoS specifications, are defined in [15]. Unsolicited grant service (UGS) and best effort (BE) are two examples of these traffic types.

To carry out traffic analysis, MCS probabilities are required from Throughput Calculation block. In [18], we have exhibited how the MCS probabilities are utilized in traffic analysis.

Before the semi-analytical method is presented, we discuss the interference model used in the simulations. 


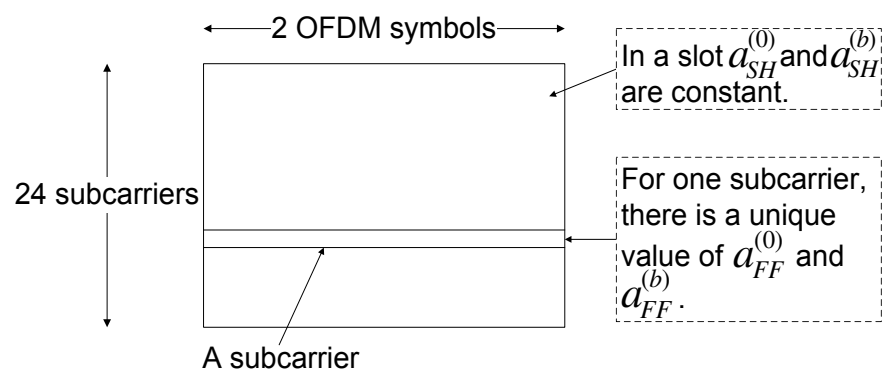

Fig. 3. Shadowing and fast fading over a PUSC slot.

\section{INTERFERENCE MODEL}

\section{A. Subcarrier SINR}

SINR of a subcarrier $n$ is given as:

$$
S I N R_{n}=\frac{P_{n, T x} a_{n, S h}^{(0)} a_{n, F F}^{(0)} \frac{K}{d^{(0)^{\alpha}}}}{N_{0} W_{S c}+\sum_{b=1}^{B} P_{n, T x} a_{n, S h}^{(b)} a_{n, F F}^{(b)} \frac{K}{d^{(b)^{\alpha}}}},
$$

where $P_{n, T x}$ is the per subcarrier power, $a_{n, S h}^{(0)}$ and $a_{n, F F}^{(0)}$ represent the shadowing (log-normal) and fast fading (Rayleigh) factors for the signal received from serving BS respectively, $B$ is the number of interfering $\mathrm{BS}, K$ is the path loss constant, $\alpha$ is the path loss exponent and $d^{(0)}$ is the distance between MS and serving BS. The terms with superscript $b$ are related to interfering BS. $W_{S c}$ is the subcarrier frequency spacing and $N_{0}$ is the thermal noise density. The values of pathloss constant and exponent are derived from COST231 Hata macrourban path loss model [15].

\section{B. Effective SINR}

We compute $S I N R_{\text {eff }}$ over the subcarriers of a slot. The physical abstraction model used for this purpose is mean instantaneous capacaity (MIC) [15]. For computation of $S I N R_{e f f}$, log-normal shadowing is drawn randomly for a slot and is same for all subcarriers of a slot. Since subcarriers of a subchannel (hence a slot) are not contiguous, fast fading is drawn independently for every subcarrier of a slot (Fig. 3). For fast fading, Rayleigh distribution has been considered in simulations.

\section{Semi-Analytical Method}

A systematic overview of the proposed semi-analytical method is depicted in Fig. 4. The method is divided into two steps: A) Simulations and Distribution/Curve Fitting and B) Off-line Application. In the following text, these steps are explained in detail.

\section{A. Simulations and Distribution/Curve Fitting}

During this step, spatial distributions of $S I N R_{\text {eff }}$ is obtained using Monte Carlo simulations for a given value of $R, P_{T x}$ and a specified range of $\sigma_{S H}$ integral values. The parameters (mainly based on [15]) can be found in Tab. II. The details of simulator can be found in [16].

Each distribution of $S I N R_{e f f}$ is specific to a value of $\sigma_{S H}$. With the help of distribution fit (based on maximum likelihood estimation), the parameters of GEV distribution
TABLE II

PARAMETERS AND DETAILS OF SIMULATIONS.

\begin{tabular}{|l|l|}
\hline Parameter & Value \\
\hline Reuse type & $3 \times 3 \times 3$ \\
\hline No. of interfering BS & 18 using wraparound technique \\
\hline Spatial distribution of MS & Uniform random \\
\hline Number of MS dropped per sector & 1 \\
\hline Number of snapshots & 10000 \\
\hline Carrier frequency $f_{c}$ & $2.5 \mathrm{GHz}$ \\
\hline Subcarrier spacing $\triangle f$ & $10.9375 \mathrm{kHz}$ \\
\hline TDD frame duration & $5 \mathrm{~ms}$ \\
\hline Thermal noise density $N_{0}$ & $-174 \mathrm{dBm} / \mathrm{Hz}$ \\
\hline Shadowing standard deviation $\sigma_{S H}$ & $8.9 \mathrm{~dB}$ \\
\hline Height of BS $h_{B S}$ & $32 \mathrm{~m}$ \\
\hline Height of MS $h_{M S}$ & $1.5 \mathrm{~m}$ \\
\hline $\begin{array}{l}\text { Antenna beam pattern } 3 \mathrm{GPP} 2 \\
G(\psi), \text { where } \psi \text { is the angle MS }\end{array}$ & \multicolumn{2}{|c|}{${ }_{\max }+\mathrm{max}$} \\
subtends with sector boresight & \\
\hline Antenna Gain (boresight) $G_{\max }$ & $16 \mathrm{dBi}\left(\frac{\psi}{\psi_{3 d B}}\right)^{2},-G_{F B}$ \\
\hline Front-to-back power ratio $G_{F B}$ & $25 \mathrm{~dB}$ \\
\hline 3-dB beamwidth $\psi_{3 d B}$ & $70^{\circ}$ \\
\hline
\end{tabular}
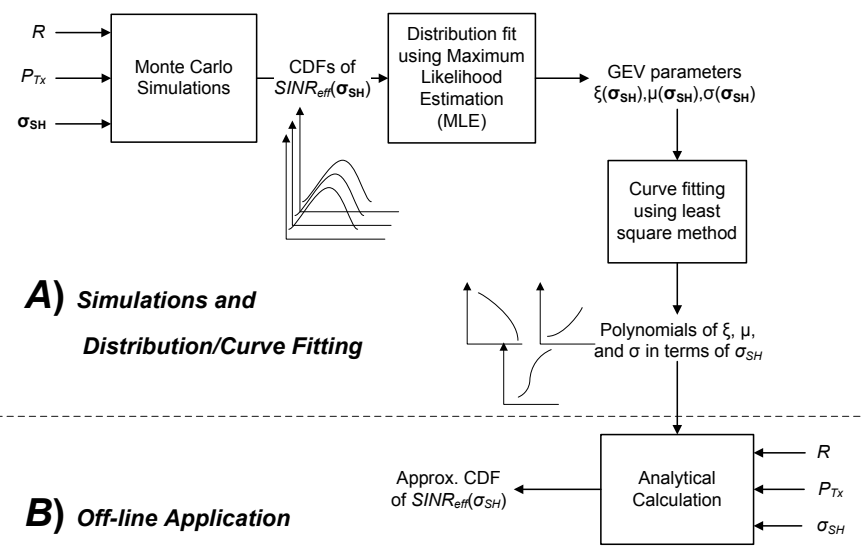

Fig. 4. Overview of proposed semi-analytical method.

(shape parameter $\xi$, scale parameter $\sigma$ and location parameter $\mu$ ), approximating the simulation PDFs, are acquired for each value of $\sigma_{S H}$.

In order to evaluate the distribution fit, the dissimilarity or error $\Xi$ between GEV and simulation PDFs, $\varphi_{G E V}$ and $\varphi_{\text {sim }}$, is quantified as follows [19]:

$$
\Xi \triangleq \int_{-\infty}^{\infty}\left|\varphi_{G E V}(t)-\varphi_{\text {sim }}(t)\right| d t
$$

Since the area under a PDF is 1 , the maximum value of error can be 2 . Hence the value of error can be between 0 and 2 i.e., $0 \leq \Xi \leq 2$.

Once it is verified that simulation PDFs of $S I N R_{\text {eff }}$ can be approximated by GEV PDFs, three GEV parameters are then separately plotted against the integral values of $\sigma_{S H}$. With the help of curve fitting (using least square method), distinct polynomials, expressing each parameter in terms of $\sigma_{S H}$, are found.

\section{B. Off-line Application}

To calculate $S I N R_{\text {eff }}$ distribution for any desired value (integral/non-integral) of $\sigma_{S H}$ in the range specified in section IV-A, we no longer require to carry out time consuming Monte 
Carlo simulations. It is sufficient to find out GEV parameters through polynomials for that value of $\sigma_{S H}$. Then using GEV $\mathrm{CDF}$ and thresholds values of $S I N R_{e f f}$ for different MCS types of Tab. I, probabilities of these MCS can be obtained. These MCS probabilities are used to calculate sector/cell throughput by applying Eq. 1. In section $\mathrm{V}$, we also show that results obtained through this method are applicable for various values of $R$ and $P_{T x}$.

\section{NUMERICAL RESULTS}

In this section, we present the numerical results. For Monte Carlo simulations, range of $\sigma_{S H}$ is considered to be $4,5, \ldots, 12$. Other input parameters are $R=1500 \mathrm{~m}$ and $P_{T x}=43 \mathrm{dBm}$. An $S I N R_{\text {eff }}$ distribution is obtained for each value of $\sigma_{S H}$. Using distribution fitting, GEV parameters are determined for each of these distributions. As an example, in Fig. 5, approximation of $S I N R_{e f f}$ PDF (obtained through simulation) by a GEV PDF for $\sigma_{S H}=9 \mathrm{~dB}$ is shown. As can be noticed, the two distributions only have a dissimilarity error of 0.052 which is $2.6 \%$ of the maximum possible error.

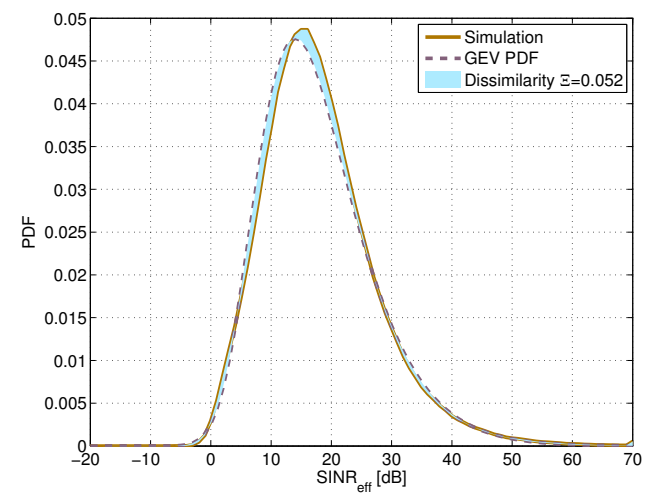

Fig. 5. $S I N R_{e f f}$ distribution through simulation and GEV polynomial for $\sigma_{S H}=9 \mathrm{~dB}, R=1500 \mathrm{~m}, P_{T x}=43 \mathrm{dBm}$ and reuse $3 \times 3 \times 3$.

GEV parameters, obtained though distribution fitting, are separately plotted against $\sigma_{S H}$ values in Figs. 6, 7 and 8 . With the help of curve fitting, polynomials of the curves approximating these plots are found and are also given in the figures. As can be noted in the figures that the degree of all polynomials never exceeds four. These polynomials can instantaneously give values of GEV parameters for any value of $\sigma_{S H}$.

To validate off-line application (cf. section IV-B), we choose an arbitrary value $\sigma_{S H}=7.5 \mathrm{~dB}$. We calculate the GEV parameters through polynomials and get PDF, MCS probabilities and cell throughput. For the same value of $\sigma_{S H}$ and assuming the values of $R=1500 \mathrm{~m}, P_{T x}=43 \mathrm{dBm}$, we find the PDFs, MCS probabilities and cell throughput through simulations. Furthermore, we also check the applicability of results obtained through GEV parameters, with $\sigma_{S H}=7.5 \mathrm{~dB}$, for various cell configurations. For this purpose, we fix $\sigma_{S H}=$ $7.5 \mathrm{~dB}$ and carry out simulations for different values of $R$ and $P_{T x}$. The maximum value of $R$ is considered to be $2000 \mathrm{~m}$ beyond which outage probability increases rapidly [20]. PDFs, MCS probabilities and average cell throughput are obtained

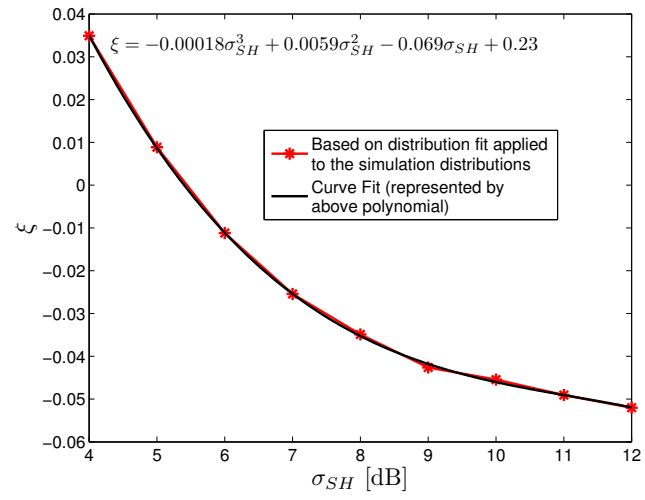

Fig. 6. Shape parameter $\xi$ of GEV distribution versus $\sigma_{S H}$ for $R=1500 \mathrm{~m}$, $P_{T x}=43 \mathrm{dBm}$ and reuse $3 \times 3 \times 3$.

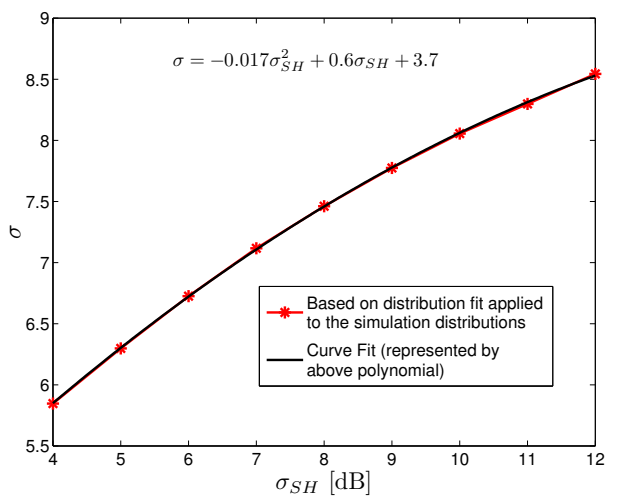

Fig. 7. Scale parameter $\sigma$ of GEV distribution versus $\sigma_{S H}$ for $R=1500 \mathrm{~m}$, $P_{T x}=43 \mathrm{dBm}$ and reuse $3 \times 3 \times 3$.

through simulations with different configurations are compared with those obtained through GEV parameters.

The results of validation and applicability for various cell configurations are given in Fig. 9 and Tab. III. For MCS probabilities, maximum difference was found to be 0.06 (for MCS 64QAM-3/4) with simulation configuration of $R=1000 \mathrm{~m}$, $P_{T x}=43 \mathrm{dBm}$, which is $13 \%$ of the value of MCS 64QAM$3 / 4$ probability. As far as cell throughput and PDF error are concerned, the percentage error w.r.t maximum possible error never exceeds $5 \%$ and cell throughput does not differ more than $5.47 \%$ for all cell configurations.

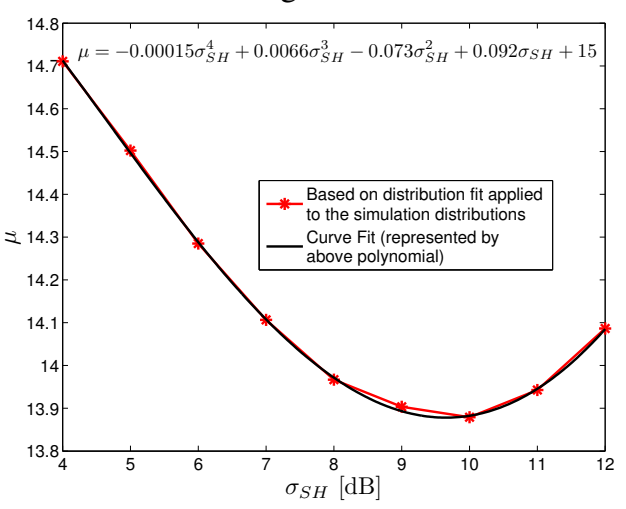

Fig. 8. Location parameter $\mu$ of GEV distribution versus $\sigma_{S H}$ for $R=$ $1500 \mathrm{~m}, P_{T x}=43 \mathrm{dBm}$ and reuse $3 \times 3 \times 3$. 
TABLE III

COMPARISON OF RESULTS OBTAINED THROUGH SIMULATION AND GEV PARAMETERS FOR $\sigma_{S H}=7.5 \mathrm{DB}$

\begin{tabular}{|c|c|c|c|c|c|c|}
\hline \multicolumn{2}{|c|}{$\begin{array}{l}\text { Simulation } \\
\text { Configuration }\end{array}$} & \multirow{2}{*}{$\begin{array}{l}\text { Dis- } \\
\text { simi- } \\
\text { larity } \\
\Xi\end{array}$} & \multirow{2}{*}{$\begin{array}{l}\text { Perc- } \\
\text { entage } \\
\text { w.r.t } \\
\text { max } \\
\text { error }\end{array}$} & \multicolumn{2}{|c|}{$\begin{array}{l}\text { Throughput } \\
\text { X } \\
\text { [Mbps] }\end{array}$} & \multirow[t]{2}{*}{$\begin{array}{l}\text { Percentage } \\
\text { difference }\end{array}$} \\
\hline $\mathbf{P}_{\mathbf{T x}[\mathrm{dBm}]}$ & $\overline{\mathbf{R}_{[\mathrm{m}]}}$ & & & Sim & GEV & \\
\hline 43 & 1000 & 0.095 & 4.73 & 5 & 4.73 & 5.47 \\
\hline 43 & 1250 & 0.073 & 3.65 & 4.96 & 4.73 & 4.57 \\
\hline 43 & 1500 & 0.056 & 2.83 & 4.88 & 4.73 & 3.17 \\
\hline 43 & 1750 & 0.058 & 2.92 & 4.78 & 4.73 & 1.18 \\
\hline 43 & 2000 & 0.1 & 5 & 4.66 & 4.73 & 1.35 \\
\hline$\overline{40}$ & 1500 & 0.065 & 3.27 & 4.75 & 4.73 & 0.49 \\
\hline 46 & 1500 & 0.075 & 3.77 & 4.96 & 4.73 & 4.72 \\
\hline
\end{tabular}

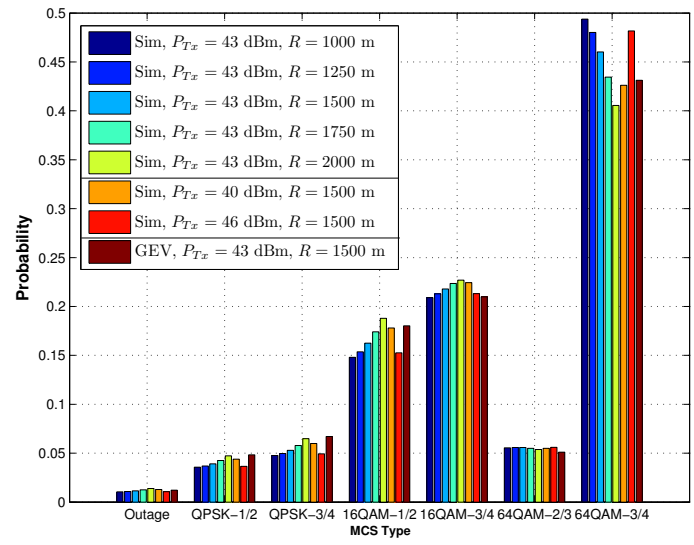

Fig. 9. MCS Probabilities for $\sigma_{S H}=7.5 \mathrm{~dB}$ and reuse $3 \times 3 \times 3$.

The simulations were run on a computer with following specifications: $3 \mathrm{GHz}$ Intel Core 2 Duo processor, $2 \mathrm{~GB}$ RAM and 4 MB shared L2 cache. Time taken by one Monte Carlo simulation was about 5 hours. Time required for semianalytical method is around $N_{S H} \times 5$ hours, where $N_{S H}$ is the length of vector $\sigma_{\mathbf{S H}}$. If MCS distributions are required for $N$ different scenarios (each defined by specific values of $\sigma_{S H}, R$ and $P_{T x}$ ), our proposed method always requires fixed duration which is equal to $N_{S H} \times 5$ hours while the same task carried out by Monte Carlo simulations will require $N \times 5$ hours.

\section{CONCLUSION}

In this paper, we have proposed a semi-analytical method to model SINR statistics in mobile WiMAX cellular networks. We have shown that $S I N R_{e f f}$ distribution, obtained through system level Monte Carlo simulations, can be successfully approximated by a GEV distribution. It is further illustrated that the parameters of GEV distribution can be expressed using simple polynomials in terms of $\sigma_{S H}$. These polynomials can be used to calculate the GEV parameters for any desired value of $\sigma_{S H}$. These parameters can be used to estimate $S I N R_{e f f}$ distribution and hence the MCS stationary probabilities. The results can be used for a number of network configurations with sufficient accuracy. As a result, we no longer require exhaustive simulations to derive distribution of $S I N R_{e f f}$.

\section{ACKNOWLEDGMENT}

This work was partially sponsored by Alcatel-Lucent Bell Labs France.

\section{REFERENCES}

[1] "IEEE Standard for Local and Metropolitan Area Networks, Part 16: Air Interface for Fixed and Mobile Broadband Wireless Access Systems, Amendment 2: Physical and Medium Access Control Layers for Combined Fixed and Mobile Operation in Licensed Bands and Corrigendum $1, " 2005$.

[2] M. Zorzi, "On the Analytical Computation of the Interference Statistics with Applications to the Performance Evaluation of Mobile Radio Systems," in Trans. of IEEE on Communications, Jan 1997.

[3] J. Cho and D. Hong, "Statistical Model of Downlink Interference for the Performance Evaluation of CDMA Systems," in IEEE Communications Letters, Nov 2002.

[4] A. Mäder and D. Staehle, Interference Estimation for the HSDPA Service in Heterogeneous UMTS Networks. Book Chapter in "Lecture notes in computer science", Springer Publications Berlin, Germany, 2006.

[5] C. Ball, E. Humburg, K. Ivanov, and F. Treml, "Performance Analysis of IEEE802.16 Based Cellular MAN with OFDM-256 in Mobile Scenarios," in Proc. of IEEE VTC Spring, May 2005.

[6] C. Ball, E. Humburg, K. Ivanov, and R. Müllner, "Rapid Estimation Method for Data Capacity and Spectrum Efficiency in Cellular Networks," in Proc. of 14th IST Mobile and Wireless Communications Summit, Jun 2005.

[7] H. Jia, Z. Zhang, G. Yu, P. Cheng, and S. Li, "On the Performance of IEEE 802.16 OFDMA System under Different Frequency Reuse and Subcarrier Permutation Patterns," in Proc. of IEEE ICC, Jun 2007, pp. 5720-5725.

[8] M. Einhaus, O. Klein, B. Walke, and R. Halfmann, "MAC Level Performance Comparison of Distributed and Adjacent OFDMA Subchannels in IEEE 802.16," in Proc. of European Wireless (EW), Apr 2007.

[9] Y.-J. Choi, C. S. Kim, and S. Bahk, "Flexible Design of Frequency Reuse Factor in OFDMA Cellular Networks," in Proc. of IEEE ICC, Jun 2006.

[10] J.-W. So, "Performance Analysis of VoIP Services in the IEEE 802.16e OFDMA System With Inband Signaling," in Trans. of IEEE on Vehicular Technology, May 2008.

[11] S. K. Kim and C. G. Kang, "Throughput Analysis of Band AMC Scheme in Broadband Wireless OFDMA System," in Proc. of IEEE WCNC, Apr 2006.

[12] C. Monti, R. Giuliano, F. Mazzenga, and P. Loreti, "Interference Evaluation for a Cellular System Based on OFDMA," in Proc. of Second International Mobile Multimedia, Sep 2006.

[13] S.-E. Elayoubi, B. Fourestié, and X. Auffret, "On the Capacity of OFDMA 802.16 Systems," in Proc. of IEEE ICC, Jun 2006.

[14] S. Markose and A. Alentorn, "The Generalized Extreme Value (GEV) Distribution, Implied Tail Index and Option Pricing," University of Essex, Department of Economics, Economics Discussion Papers 594, Apr. 2005. [Online]. Available: http://ideas.repec.org/p/esx/essedp/594. html

[15] K. Ramadas and R. Jain, "WiMAX System Evaluation Methodology," Wimax Forum, Jan 2007.

[16] M. Maqbool, M. Coupechoux, and P. Godlewski, "A Semi-analytical Method to Model Spatial Distribution of Effective SINR in WiMAX Networks," TELECOM ParisTech, Technical Report, Draft Version, 2008. [Online]. Available: http://perso.telecom-paristech.fr/ coupecho/ publis/rapportsemianalytic08.pdf

[17] "WiMAX Forum Mobile System Profile 4 Release 1.0 Approved Specification," Wimax Forum, May 2007.

[18] G. Nogueira, B. Baynat, M. Maqbool, and M. Coupechoux, Performance Evaluation and Dimensioning of WiMAX. Book Chapter in "WiMAX Networks Planning and Optimization", Auerbach Publications, CRC Press, Taylor \& Francis Group, 2008.

[19] D. Sinha, H. Zhou, and N. V. Shenoy, "Advances in Computation of the Maximum of a Set of Gaussian Random Variables," in Trans. of IEEE on Computer-Aided Design of Integrated Circuits and Systems, Aug 2007.

[20] M. Maqbool, M. Coupechoux, and P. Godlewski, "Comparative Study of Reuse Patterns for WiMAX Cellular Networks," TELECOM ParisTech, Technical Report, 2008. 